\title{
Random errors are not necessarily politically neutral ${ }^{\star}$
}

\author{
Michelle Blom ${ }^{10000-0002-0459-9917]}$, Andrew Conway ${ }^{[0000-0001-6277-2442]}$, Peter J. \\ Stuckey ${ }^{20000-0003-2186-0459]}$, Vanessa Teague ${ }^{3,4[0000-0003-2648-2565]}$, and Damjan \\ Vukcevic $^{5,6[0000-0001-7780-9586]}$ \\ 1 School of Computing and Information Systems, University of Melbourne, Parkville, Australia \\ ${ }^{2}$ Faculty of Information Technology, Monash University, Clayton, Australia \\ 3 Thinking Cybersecurity Pty. Ltd. \\ 4 College of Engineering and Computer Science, Australian National University \\ 5 School of Mathematics and Statistics, University of Melbourne, Parkville, Australia \\ 6 Melbourne Integrative Genomics, University of Melbourne, Parkville, Australia
}

\begin{abstract}
Errors are inevitable in the implementation of any complex process. Here we examine the effect of random errors on Single Transferable Vote (STV) elections, a common approach to deciding multi-seat elections. It is usually expected that random errors should have nearly equal effects on all candidates, and thus be fair. We find to the contrary that random errors can introduce systematic bias into election results. This is because, even if the errors are random, votes for different candidates occur in different patterns that are affected differently by random errors. In the STV context, the most important effect of random errors is to invalidate the ballot. This removes far more votes for those candidates whose supporters tend to list a lot of preferences, because their ballots are much more likely to be invalidated by random error. Different validity rules for different voting styles mean that errors are much more likely to penalise some types of votes than others. For close elections this systematic bias can change the result of the election.
\end{abstract}

\section{Introduction}

We investigate the effects of random errors on election outcomes, in the context of preferential elections counted using the Single Transferable Vote (STV). It is often assumed that random errors (whether from human or manual counting) are unimportant because they are likely to have nearly equal effects on all candidates. In this paper we show that this is not the case, using simulated random errors introduced into real STV voting data. In some cases, this introduces a systematic bias against some candidates.

Random errors have a non-random effect because real votes are not random. Voters not only express different preferences, but express them in a different way, according to whom they choose to support.

In STV, some candidates are elected mainly on the strength of their party listing; others rely on gathering preference flows from other parties, or on their individual popularity relative to their party's other candidates. So when we look at the votes that contributed

${ }^{\star}$ A later version of this work appears in E-Vote-ID 2020. 
to the election of different candidates, we find that the types of votes chosen by their supporters may be very different. Hence a random error that affects different types of votes differently introduces a systemic change in the election result.

One obvious kind of error is to misrecord a number. Usually, this either invalidates the ballot completely, or invalidates preferences below the error. The more preferences there are on a ballot, the more likely that at least one of them is misrecorded. So as a general rule, candidates that are more dependent on later preferences or long preference lists are more severely disadvantaged by random errors.

Although these results are significant, and need to be taken into account for close contests, we find that reasonable error rates produce changes in only very few elections, which (so far) correspond only to those that are obviously very close. It is possible for STV elections to have hidden small margins, but this seems to be uncommon-in almost all the elections we simulated, no plausible error rate produced a change in outcome. Typical random error rates will affect election results when the election is close, but are not expected to do so when the election is not close.

We do not consider the errors necessary to alter the election result in a targeted way by altering specific carefully chosen votes-they would obviously be much smaller. Hence the results of this paper apply to random errors, but not deliberate electoral fraud.

The remainder of the paper is organized as follows. In the next section we explain STV elections, in particular in the case of Australian Senate elections, and discuss how the votes are digitised and counted. In Section 3 we describe our experiment design and introduce the three error models we explore. In Section 4 we provide a number of different approaches to estimate the likely error rate that occurs for Australian Senate elections. In Section 5 we examine the result of applying simulated errors to Australian Senate elections and discuss how these errors can change the result of the election. Finally in Section 6 we conclude.

\section{Background on STV counting}

\subsection{The Single Transferable Vote (STV) counting algorithm}

STV is a multi-winner preferential voting system. Candidates compete for $s$ available seats. A candidate is awarded a seat when their tally reaches or exceeds the quota, $Q$, defined as a function of the number of ballots cast in the election, $|\mathcal{B}|$, and the number of seats, $s$. One popular definition is the Droop quota,

$$
Q=\left\lfloor\frac{|\mathcal{B}|}{s+1}\right\rfloor+1 .
$$

When a voter casts a ballot in one of these STV elections, they have the option of voting 'above the line' or 'below the line'. Figure 1 shows an example of a ballot for a simple STV election in which candidates from three parties are competing for $s$ seats. Each 


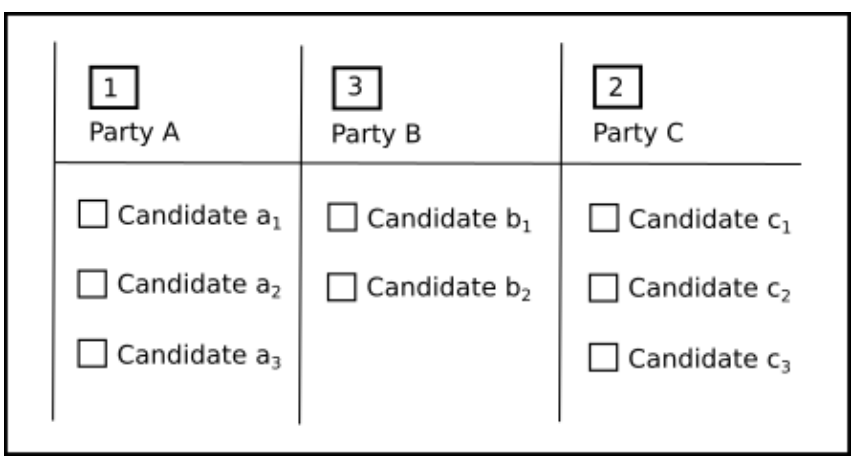

(a)

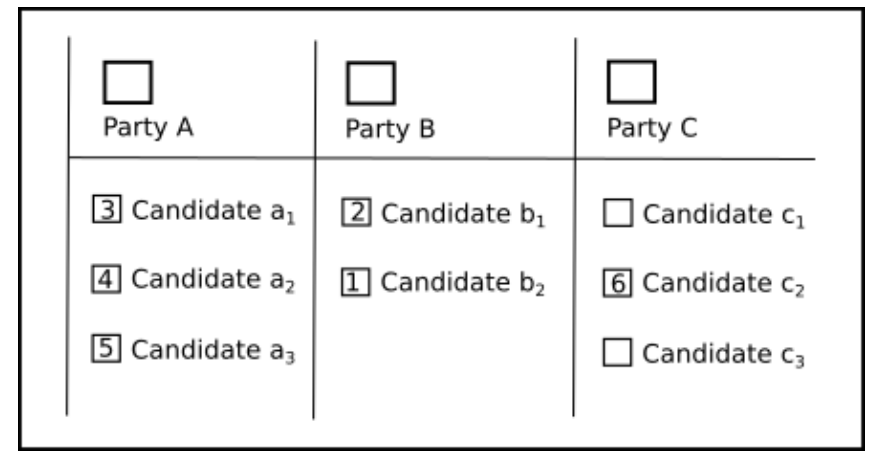

(b)

Fig. 1: An example of two simple ballots for a 3-party STV election. In (a), the voter has chosen to vote above the line, and in (b) they have voted below the line.

party or group of independents fielding candidates in the election have a box sitting 'above the line' (ATL). A voter may rank these parties and groups by placing a number in their corresponding box (Figure 1a). Alternatively, a voter may rank individual candidates by placing a number in their box, below the line (BTL) (Figure 1b).

Tabulation starts by giving each candidate all the below-the-line ballots in which they have been ranked first. ATL ballots are awarded to the first candidate listed under the party that has been ranked first. For example, a ballot in which Party A has been ranked first sits in the first preference pile of candidate $a_{1}$. A BTL ballot in which candidate $b_{2}$ is ranked first sits in that candidate's first preference pile. Each ballot is assigned a weight, starting at 1 , that changes as counting proceeds. The tally of a candidate is the sum of the weights of ballots sitting in their tally pile, possibly with some rounding.

Counting proceeds by awarding a seat to all candidates whose tallies have reached or exceeded $Q$. Their surplus - their tally after subtracting $Q$-is distributed to remaining eligible candidates. A candidate is eligible if they have not been eliminated, and their tally has not reached a quota's worth of votes. The ballots sitting in an elected candi- 
date's tally pile are re-weighted so that their combined weight is equal to the candidate's surplus. These ballots are then given to the next most-preferred eligible candidate on their ranking. The ATL ballot in Figure 1a is given to candidate $a_{2}$ if $a_{1}$ is elected to a seat. If neither $a_{2}$ or $a_{3}$ are eligible, the ballot then moves to candidate $c_{1}$. The BTL ballot in Figure $1 \mathrm{~b}$ is given to candidate $b_{1}$ if $b_{2}$ is elected or eliminated.

If no candidate has reached a quota, the candidate with the smallest tally is eliminated. The ballots in their tally pile are distributed to the next most-preferred eligible candidate in their rankings at their current weight.

Counting proceeds in rounds of election and elimination until all $s$ seats are filled, or until the number of candidates that remain eligible equals the number of remaining seats. In this setting, each of the remaining candidates is awarded a seat.

\subsection{Australian vote digitisation in practice}

Australians cast their votes on paper ballots. The Australian Electoral Commission (AEC) digitises the preferences in a hybrid manual and automated process. Precise details about this process are unavailable, but most ballots seem to receive both automated digitisation and secondary human data entry. (Ballots that are judged blank are not re-examined.) It is possible that manual data entry is performed on ballot papers. ${ }^{7}$ Other pamphlets suggest that only the images, not the paper ballots, are used. ${ }^{8}$

An automated system then checks, for each ballot, whether the automated digitisation matches the human interpretation. Obviously this does not defend against software errors or deliberate manipulation, particularly downstream of the process, but it probably does produce reasonably low random error rates, assuming that the human errors are not highly correlated with the errors of the automated system.

Ballots are required to have a minimum number of preferences before they are considered valid; such ballots are referred to as formal ballots. In the 2016 and 2019 elections, a BTL formal vote must have every preference from 1 to 6 inclusive present exactly once; an ATL formal vote requires the preference 1 to be present exactly once and a formal BTL vote not to be present. According to the information about the digitisation processes mentioned above, non-blank informal ballots seem to get a second human inspection automatically.

The AEC publishes on their website the complete digitised preferences for all Senate votes, excluding blanks and votes judged to be informal.

In summary, the published data could differ from the actual ballots for many reasons:

- random errors that match in both the automated and human digitisation process,

- random errors that occur in either the automated or human digitisation process, and are endorsed rather than corrected by the reconciliation process,

\footnotetext{
${ }^{7}$ https://www.aec.gov.au/Voting/counting/files/css-integrity.pdf

${ }^{8}$ https://www.aec.gov.au/Voting/counting/files/senate-count.pdf
} 
- erroneous exclusion of ballot papers judged to be informal,

- accidental alterations, duplicates or omissions caused by software bugs,

- deliberate manipulation by malicious actors, either of the images (before digitisation) or of the preference data (from digitisation to publication).

Our investigation does not apply to the last two kinds of errors, which could be introduced in a non-random way that worked for or against a particular candidate. It does apply to the errors that are random. In particular, we show that digitisation errors that randomly cause some ballots to be judged informal can impact candidates differently.

\section{Experimental design}

Our analysis is performed on the AEC's published data for the 2016 and 2019 Australian federal elections for the Senate, i.e. the output of the process described in Section 2.2. Ideally our analysis would be based upon the actual marks that voters made on their ballots, or even what they intended to make, and the comparison with the AEC's output. However, these data are not available. Instead, we use the AEC's output as the 'actual' ballot data, and add simulated errors.

\subsection{Analysis code}

For logistical reasons, and to make it easy for anyone to replicate this experiment, we extract those preferences that are actually considered valid in the election. If a number is absent or repeated in the preference marks, then it and all subsequent preferences are disregarded. We have made available a standardised "stv" file format based on the data published by the $\mathrm{AEC}^{9}$. This common format does unfortunately mean that we lose some (invalid) marks that could conceivably have become valid when we added new random errors, or which could, through errors, invalidate earlier preferences.

We used the Java pseudo-random number generator java. ut il. Random to generate random numbers, and ensured that different executions used different seeds. Our code is available for download ${ }^{10}$.

\subsection{Error models}

We simulate the effect of errors by making random changes to the votes. We are not certain exactly what "random" failures in the scanning process would be, so we have devised three different models for simulated errors, in increasing order of complexity and plausibility. The first models an error where, somewhere in the list, something goes

\footnotetext{
${ }^{9}$ See the downloads section for each election at: https://vote. andrewconway.org

${ }^{10}$ https://github.com/SiliconEconometrics/Publicservice
} 
wrong that invalidates the rest of the preference list. The second models an error in which a digit is randomly misread as another digit, chosen uniformly. The final model recognises that some misreadings are much more likely than others-for example, a 3 is more likely to be confused with an 8 than a 1 - so we use a model that includes a specific error probability for each digit and each potential misreading.

Each model applies to a valid list of preferences and treats either each number or each digit separately with random errors chosen independently.

1. For each preference, with probability $\epsilon$, truncate the list at that preference.

2. For each digit, with probability $\epsilon$, replace that digit with a digit uniformly chosen from $\{0,1,2,3,4,5,6,7,8,9\}$, which may be the original digit.

3. Start with a table of pairwise error ratios for digits such as Table 1 (that is, the probability that a certain digit is mistranscribed into a certain other digit). For each digit, change it into a different digit with the probability given in the table.

Note that in all three models, the probability of at least one error on the ballot increases with the number of preferences listed on the ballot. We are primarily motivated by machine errors, so per-digit or per-number random errors seem plausible, but it is worth noting that other errors might be important too, such as models that considered that some voters (those with bad handwriting) were much more likely to have their vote misinterpreted than others.

After applying errors, formality rules are checked again, reducing the number of ballots considered for the election.

\section{What is a realistic error rate?}

As far as we know, there are no publicly available results from any rigorous estimate of Senate scanning random errors in Australia. However, there are several independent estimates, which give us a per-digit error rate ranging from $0.01 \%$ to $0.38 \%$. We define an error to be a discrepancy between the paper ballot and the electronic preference list output at the end of the process.

\subsection{Using data from the Australian Electoral Commission}

As far as we know, the AEC does not conduct, nor allow anyone else to conduct, a large random sample of Senate ballots for comparison between electronic and paper records. However, an Australian National Audit Office report ${ }^{11}$ describes a process for gaining an estimate from a small sample. This process was conducted by AEC officials.

\footnotetext{
11 https://www.anao.gov.au/work/performance-audit/ aec-procurement-services-conduct-2016-federal-election
} 
- A batch of 50 ballot papers was randomly selected and then six ballot papers from that batch were reviewed;

- Compliance inspectors recorded the first six preferences from the physical ballot paper on a checklist;

- Verification officers compared the preferences recorded on the checklist against those on the scanned image of the ballot paper and those in the related XML file;

- The IT security team compiled, investigated and reported on the findings.

The compliance inspection report outlined that a total of 1,510 ballot papers were inspected and 4 processing errors were identified. This seems to indicate an error rate of less than $0.3 \%$ per ballot. Although it wasn't recorded how many preferences were on each ballot, it seems to indicate a very small per-digit error rate. However, a careful reading of that experimental description shows that the officials verified only the numbers from 1 to 6 . Errors in later preferences were ignored. So this estimate may substantially underestimate the overall rate of error.

To estimate the per-digit error rate implied by these data, we assumed that all of the 1,510 ballot papers that were inspected had six preferences marked on them, giving a total of 9,060 digits. We also assumed that the 4 'processing errors' were each a singledigit error. This gave a per-digit error rate of $0.04 \%$, with a $95 \%$ confidence interval of $(0.01 \%, 0.11 \%)$.

In reality, some proportion of these ballot papers were likely to be informal and have fewer than six preferences marked. Adjusting the above assumptions based on reported rates of informality by the $\mathrm{AEC}^{12}$ had negligible impact on these estimates.

\subsection{Informal experiment}

For the 2019 federal election, we conducted an informal experiment amongst 15 of our colleagues to get a rough estimate of the 'end to end' accuracy of the Senate vote digitisation process. Each of our colleagues decided on their Senate vote ahead of the election and made a private record of it for later comparison. On polling day, they each carefully completed their Senate ballot paper in accordance with their planned vote. After the election, it was possible to compare these against the electronic file of ballots published by the AEC. Each of our colleagues searched for a vote that matched their own vote either exactly or very closely.

All of our colleagues voted below the line in Victoria. Due to the very large number of possible ways to vote below the line, each of their votes was extremely likely to be unique. In addition, the electronic file from the AEC also recorded the polling booth for each ballot. These two facts together allowed each of our colleagues to easily identify their own ballot paper in the file and be confident that it was indeed their own. This was true even if the match were not exact, since the next 'closest' matching ballot would typically vary substantially from each person's private record.

\footnotetext{
${ }^{12}$ For example, https://www.aec.gov.au/Voting/Informal_Voting/senate/
} 
Of our 15 colleagues, 12 found ballots in the file that exactly matched their own records. This indicates perfectly accurate digitisation. The remaining 3 found a mismatch: each of them had a single one of their preferences recorded differently in the file than in their private record. These mismatches could be due to an error in the AEC digitisation process or to a transcription error on the part of our colleagues. However, they do give us at least a rough estimate of accuracy.

What per-digit error rate does this imply? We use the following assumptions: a) Each ballot had votes below the line; b) All boxes below the line were numbered; c) All of the reported errors were for a single digit. These assumptions maximise the number of possible digits and minimise the number of errors, and thus will give the lowest possible error rate estimate. There were 82 candidates for Victoria. This gives $9+73 \times 2=155$ digits per ballot, which is $155 \times 15=2,325$ digits in total. Out of these, we have 3 single-digit errors. These give a per-digit error rate of $0.13 \%$, and a $95 \%$ confidence interval of $(0.03 \%, 0.38 \%)$. The error rate here captures any errors either by a voter or by the digitisation process, so it provides a rough upper bound on the latter's error rate.

\subsection{What is the state of the art in digit recognition error rate?}

Accurately recognizing handwritten digits by computer is an important consideration for many applications where data crosses from the physical world into the digital. The MNIST (Modified National Institute of Standards and Technology) database is a large database of handwritten digits that is commonly used for training image processing systems. The database consists of digits written by high school students and American Census Bureau employees, and normalised to be represented as grayscale images of size $28 \times 28$ pixels. The current state of the art approach [1] to this dataset has an error rate of $0.18 \%{ }^{13}$ Care must be taken with this result, which is on a well studied and well curated data set. While Australian ballot papers have boxes marked where each number should be filled in, not all digits written in practice fall completely within the box. Nevertheless, this gives an accurate lower bound on pure computer-based digit recognition accuracy. The AEC process involves human inspection which means that it may be able to achieve better overall digit recognition accuracy.

The errors in digit recognition are not uniform: some digits are easier to confuse, for example 1 and 7. Most work on digit recognition does not publish the cross-digit confusion rates. Table 1 gives a confusion table showing the percentage of each actual digit versus its predicted value from experiments reported by Toghi \& Grover [3]. The overall digit recognition error in this work is $0.89 \%$, which is substantially greater than the best results reported above.

\footnotetext{
${ }^{13}$ There is unpublished work claiming $0.17 \%$.
} 
Table 1: Pairwise error digit rates. The entry for row $x$ and column $y$ gives the percentage chance of (mis)recognizing a digit $y$ as a digit $x$. A dash ' - ' indicates less than $0.01 \%$ chance of misrecognition.

\begin{tabular}{|c|c|c|c|c|c|c|c|c|c|c|c|}
\hline & & & & & Ac & ual & & & & & \\
\hline & Digit & 0 & 1 & 2 & 3 & 4 & 5 & 6 & 7 & 8 & 9 \\
\hline & 0 & 99.22 & - & 0.08 & 0.02 & 0.10 & 0.04 & 0.14 & - & 0.06 & 0.20 \\
\hline & 1 & - & 98.75 & 0.14 & - & - & - & - & 0.40 & 0.04 & 0.08 \\
\hline & 2 & 0.12 & 0.28 & 99.56 & 0.24 & - & - & - & 0.18 & 0.02 & 0.10 \\
\hline 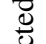 & 3 & - & - & 0.22 & 99.50 & - & - & - & 0.24 & 0.14 & 0.22 \\
\hline$\because$ & 4 & 0.16 & 0.16 & - & - & 98.65 & 0.08 & 0.10 & - & 0.12 & 0.30 \\
\hline & 5 & - & 0.02 & - & - & - & 99.52 & 0.22 & 0.10 & 0.18 & 0.12 \\
\hline & 6 & 0.10 & 0.12 & - & - & 0.06 & 0.08 & 99.48 & - & 0.14 & - \\
\hline & 7 & 0.08 & 0.42 & - & 0.16 & - & 0.02 & - & 98.90 & - & 0.38 \\
\hline & 8 & 0.10 & 0.06 & - & - & 0.48 & - & - & - & 99.16 & 0.26 \\
\hline & 9 & 0.22 & 0.20 & - & 0.08 & 0.72 & 0.26 & 0.06 & 0.18 & 0.14 & 98.34 \\
\hline
\end{tabular}

\subsection{Analysing the election data (NOT simulations) to infer the error rate}

We only have the reported ballots, not the ones that were ruled informal. (Except of course we cannot distinguish human mistakes from scanning errors.) Errors that make the vote informal are hidden.

Recall that the formality rules require at least 6 unambiguous preferences below the line, and that informal votes are not reported. We can estimate the number of hidden informal votes by observing the erroneous but formal ones. We use the number of repeated or missing numbers greater than 6 to approximate the number of repeated or missing numbers less than or equal to 6 .

Table 2 shows the data, for BTL votes cast in Tasmania for the 2016 Senate election. The first column is the preference $p$ on the ballot. The second column is the number of ballot papers that contain $p$ more than once. The final column shows the number of ballots missing that preference, showing preference $p-1$ and $p+1$ but not $p$. A 0 is not required for $p=1$. Note that there is a sudden drop at 12 because voters were instructed to list at least 12 preferences, so many people listed exactly 12 . If the 12th preference was miswritten or misrecorded, then it did not count in our table (there being no 13).

There would be no informal BTL ballots at all, and perfect zeros in the first 6 rows of Table 2, except for one special formality rule: if there is also a valid ATL vote present on the same ballot paper, then it is counted instead, and both the valid ATL vote and the invalid BTL markings are reported in the final database. Hence we expect that the numbers in the first 6 rows are only a small fraction of the ballots rendered informal by either human or scanning errors. There is a sudden increase at the 7th preference, because BTL votes with a repeated or omitted 7th preference are still included in the tally, as long as their first 6 preferences are unambiguous. 
Table 2: Counts of ballot papers with repeated and missed preferences. Tasmanian ballots with BTL marks, 2016.

\begin{tabular}{lrrrrrrrrrrrrr}
\hline Preference & 1 & 2 & 3 & 4 & 5 & 6 & 7 & 8 & 9 & 10 & 11 & 12 & 13 \\
\hline Ballots with preference repeated & 573 & 385 & 303 & 231 & 212 & 211 & 492 & 494 & 542 & 372 & 256 & 250 & 122 \\
Ballots with preference skipped & 240 & 43 & 54 & 49 & 45 & 37 & 130 & 133 & 134 & 193 & 203 & 45 & 44 \\
\hline
\end{tabular}

There are 97,685 published votes with BTL markings. Most of these were valid BTL votes but some were only published because they had valid ATL votes as well. The most representative preferences are probably 7 to 9 , being single digits whose count is not artificially suppressed due to repetitions in them causing the BTL vote to be informal and thus usually not published. For these preference numbers, the observed repetitions are on the order of $0.5 \%$. This doesn't prove that the scanning process introduces errors at a rate of $0.5 \%$ per digit, because they could be caused by voter error. It could also underestimate the scanner error rate because it includes only those not rendered informal. Nevertheless this provides an estimate of voter plus process error.

\section{Results}

\subsection{Results from truncation and digit error models}

We simulated counts with errors using the ballot data for all 8 states and territories from both the 2016 and 2019 Senate elections. We used both the truncation and digit error models, across a wide range of error probabilities. For any given choice of model and error probability, we simulated 1,000 elections (each with their own random errors under that model).

For error rates between $0 \%$ and $1 \%$, the only election for which we observed any change in the elected candidates was for Tasmania in 2016. This election was somewhat unusual in three ways. First, it was a very close election, with the difference in tallies between the final two remaining candidates, Nick McKim and Kate McCulloch, being only 141 votes. For comparison, 285 votes were lost due to rounding. Second, there was a popular labor candidate, Lisa Singh, who won a seat despite being placed fourth on the party ticket, and the candidate above her not winning a seat. This means she received many BTL votes specifically for her, rather than relying on ATL votes for the party. Finally, the 2016 election was a double dissolution, which means that twelve candidates were elected rather than the usual six.

In the real election, the 12th (final) candidate that was elected was Nick McKim. In our simulations, once we introduced a small amount of error we saw that a different candidate, Kate McCulloch, was sometimes elected instead. As we increased the perdigit error rate from $0 \%$ to $1 \%$, we saw a complete shift from one candidate to the other, see Figure 2. The truncation error model led to the same outcome (data not shown). 

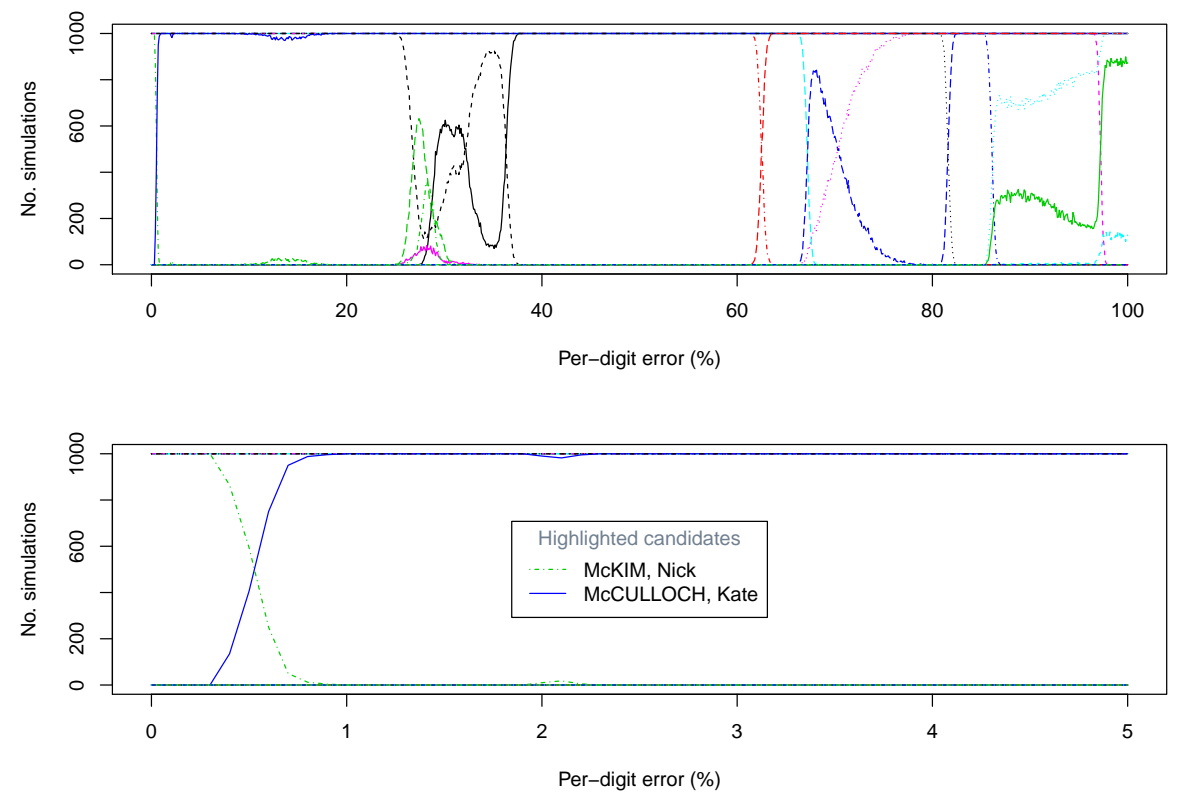

Fig. 2: Changing election outcomes as a function of error rate, Tasmanian Senate election 2016. The lower graph shows a complete reversal for a small error rate (about $0.5 \%$ ), between the state in which McKim wins consistently (no error) and that in which McCulloch wins consistently ( $1 \%$ or greater error). The upper graph shows similar behaviour for larger error rates-with error rates of more than $20 \%$ there are sharp transitions between different election outcomes.

\subsection{Pairwise digit error model}

We ran 1,000 simulations for Tasmania 2016 using the pairwise digit error model. Unlike the other models, we did not have a parameter to set but simply used the pairwise error rate matrix shown in Table 1 . This model has an average per-digit error rate of $0.89 \%$. Across the 1,000 simulations, we observed Kate McCulloch being elected $99.5 \%$ of the time, and Nick McKim for the remaining $0.5 \%$. This is consistent with the simple per-digit error model, which also resulted in Nick McKim occasionally being elected when the per-digit error was comparable.

\subsection{Sharp transitions}

The fact that such a sharp transition happens from electing one candidate to another was initially surprising to us. Rather than simply 'adding noise' and leading to randomness in which candidates got elected, the noise seems to be leading to a systematic bias in favour of or against specific candidates. This behaviour can be seen more clearly 
Table 3: Partition of the Tasmanian 2016 ballots. The number of ballots split by whether it is an above-the-line (ATL) or below-the-line (BTL) vote, and which candidate (if any) out of Kate McCulloch or Nick McKim is preferred over the other.

\begin{tabular}{lrrr}
\hline \multicolumn{4}{c}{ McCulloch McKim Neither } \\
\hline ATL & 73,975 & 97,331 & 72,468 \\
BTL & 17,066 & 42,170 & 36,149 \\
\hline
\end{tabular}

as the error rate is increased to larger values (beyond values that would be plausible in practice), see Figure 2, where sharp transitions are visible also at 28\%, 36\%, 62\%, 68\%, $82 \%, 86 \%$ and $97 \%$.

To investigate possible reasons for this, we looked at how individual ballots were affected by the simulated errors. Compared to the no-error scenario, two broad types of outcome are possible:

- The ballot becomes informal and is not counted. This will happen when it does not meet the formality requirements, e.g., does not have at least a single first preference above the line or consecutive preferences numbered 1 to 6 below the line.

- The ballot ends up exhausting before reaching a candidate. This will happen if the preference order becomes disrupted due to an error, which has the effect of truncating the preferences and not enabling the ballot to be counted in favour of any candidates further down the preference list.

We investigated these effects in the context of the Tasmanian 2016 election; we report on this in the next few sections. We found that the first type of effect was the dominant factor in determining the election outcome.

\subsection{Why random errors affect different candidates differently (Tasmania 2016)}

We saw earlier that for small error rates, we have either Nick McKim (from the Australian Greens party) or Kate McCulloch (from the One Nation Party) elected as the final candidate. There were 339,159 formal ballots for this election. For each one, we looked at the preferences to see:

- whether it was an ATL or a BTL vote,

- which of the above two candidates (or their respective parties, if it was an ATL vote) was more highly preferred, or neither one.

Table 3 shows how the ballots split into these categories. The most important fact to note is the relative number of ATL and BTL votes in favour of each candidate: more than $80 \%$ of the ballots in favour of McCulloch were ATL votes, while for McKim it was less than $70 \%$. 


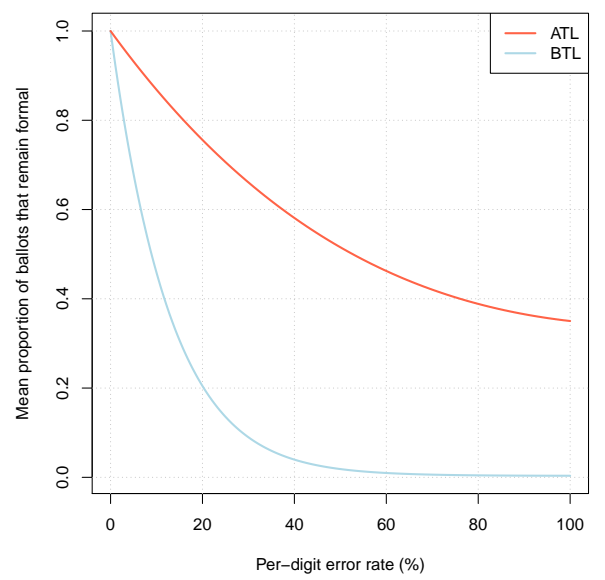

Fig. 3: Effect of the per-digit error rate on the formality of votes. The impact on above-the-line (ATL) and below-the-line (BTL) votes are shown separately.

When errors are introduced, ballots that were BTL votes were much more likely to become informal. Figure 3 illustrates this: the larger the error rate, the greater the disparity in how many of the ATL or BTL ballots became informal. This on its own is enough to explain the systematic shift from McKim to McCulloch as error rates increase.

For more insight, we took a closer look at the simulations that used a per-digit error rate of $1 \%$. For each ballot, we define the formality rate to be the proportion of simulations for which it remained formal. Figure 4 shows the distribution of the formality rate across different types of ballots. The left panel shows the clear disparity between ATL and BTL votes. This reiterates the difference we saw on average from Figure 3, but in addition we see that this disparity is very consistent across individual ballots (from the very little overlap for the ATL and BTL ballots).

When we further divided the ballots based on where in the preference list the voters placed their preferred candidate out of McKim or McCulloch, the distribution of formality rates was relatively consistent (right panel of Figure 4). This indicates that the major factor leading to McCulloch replacing McKim is simply the lower formality rate for BTL votes, after random errors were added, coupled with the fact that a larger proportion of ballots in favour of McKim were BTL votes.

For the less plausible larger errors, the sharp transitions came from new effects causing biases against major parties, who lost out as randomisation of preferences reduced their typical large first preference collection. This also caused major parties to not get multiple candidates elected in the first counting round, which meant that major party candidates low down on the party ticket tended to get eliminated before they could get preferences passed on to them, as they were reliant on BTL votes to avoid being eliminated before the first candidates of minor parties who could get ATL votes. 


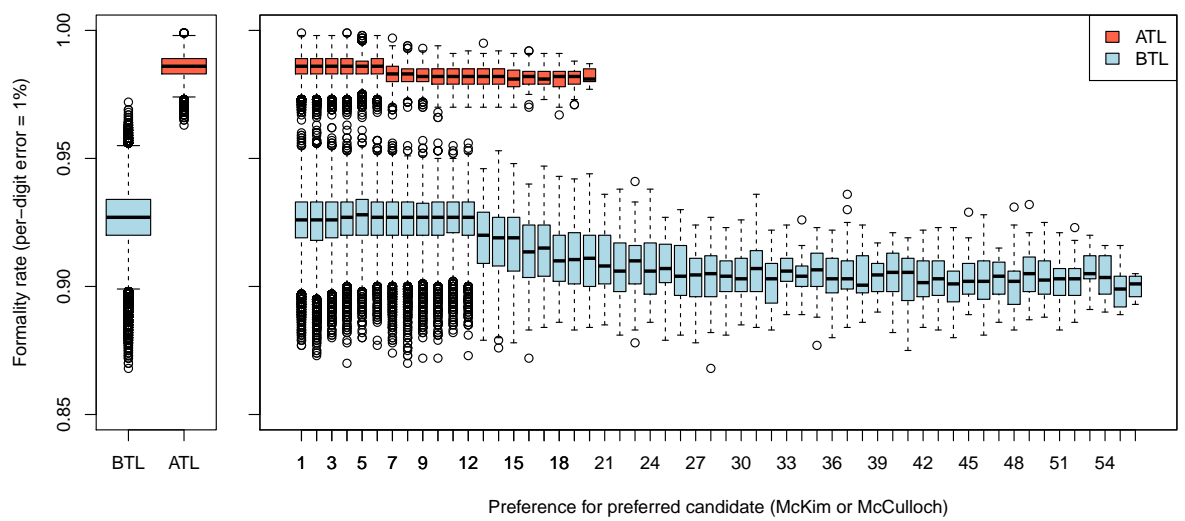

Fig. 4: Formality rates for votes with random errors injected. These are split by ATL/BTL (left panel) or by the position of the preferred candidate (right panel). Error rates vary greatly between ATL and BTL votes, but not much between preferences within those categories.

\subsection{Varying the formality requirements}

The formality requirements differ for ATL and BTL votes. In particular, BTL votes require at least 6 consecutive preferences in order to be declared formal, whereas ATL votes only require a single preference. This is one reason why the formality rate for BTL is lower once errors are introduced.

We investigated whether changing the formality rules could ameliorate the systematic bias caused by the introduction of errors. Specifically, we varied the number of consecutive preferences required for a formal BTL vote, ranging from 1 (i.e. the same as ATL votes) to 9 (i.e. more stringent than the current rules).

Figure 5 shows the impact of these choices on how often McCulloch was elected instead of McKim. Making the formality requirement less stringent reduced the bias, and once the formality rules were aligned for ATL and BTL votes, the election result remained mostly unchanged even in the presence of errors.

\subsection{Truncation of preferences}

Other than causing ballots to become informal, errors can result in votes not being counted for certain candidates if the error truncates the preference order. Candidates who obtain more of their votes from later (higher-numbered) preferences should be more affected by such truncation.

We investigated whether this might be occurring in our simulations. For each ballot, we compared the number of valid preferences before and after simulated errors. There was 


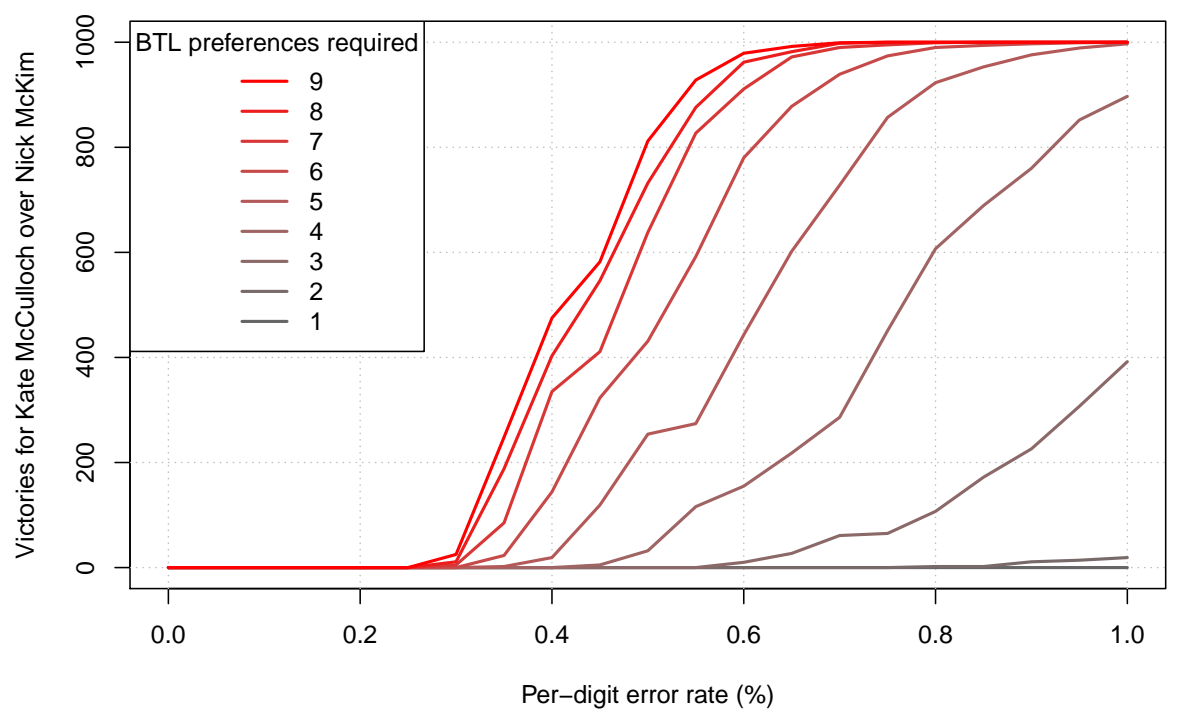

Fig. 5: The effect of formality rules on election outcomes. As the number of preferences required for a valid BTL vote increases, so does the rate at which BTL votes are excluded due to random errors. This produces a faster transition from one winning candidate to another as the error rate increases.

a clear signal of truncation: ballots that had around 60 valid preferences (which were all BTL) only had on average around 30 valid preferences remaining when the per-digit error was set to $1 \%$. In contrast, ballots that had 10 valid preferences (irrespective of whether they were ATL or BTL) maintained almost 10 valid preferences on average.

While this extent of truncation is stark, it might not necessarily lead to any change in the election outcome because many of the later preferences might not actually be used during the election count.

In the case of the Tasmanian 2016 election, we looked at ballots in favour of each of McKim and McCulloch to see whether they tended to get their votes from earlier or later preferences. Figure 6 shows the distribution of these. Interestingly, we see that McCulloch relies more on later preferences than McKim. Therefore, it is McKim rather than McCulloch that should benefit from any truncation effect. This works in the reverse direction of the formality-induced bias described earlier, however the truncation did not act strongly enough to reverse that bias.

\section{Concluding remarks}

We are not aware of any previous study of the the effects of random errors in digitization on election outcomes. While there is a considerable body of work on margin of error 

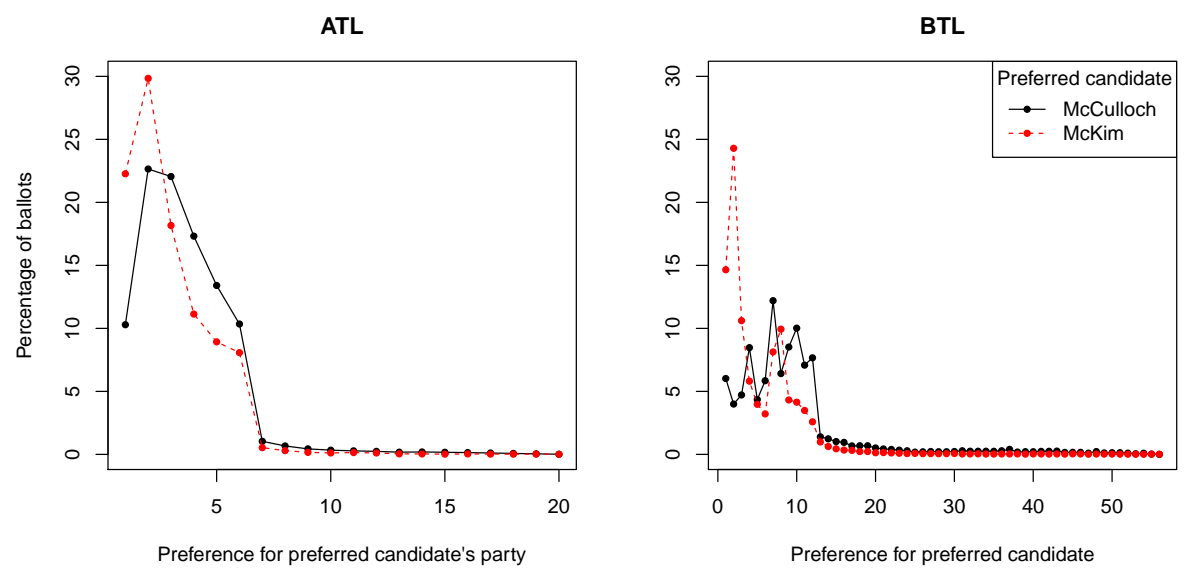

Fig. 6: Histograms of preference number. These are shown for a candidate or party depending on whether the votes are above or below the line.

for polling, there is little study of the effect of errors on elections. Richey [2] examines how 'errors' in voting can effect elections, but here the error is that a voter votes for a party that does not represent their best interests.

The previous section clearly demonstrates that random errors during counting do not necessarily lead to 'random' changes to election outcomes. We were very surprised by the sharp transitions in election results as error rates changed, illustrated in Figure 2. Systematic biases can arise due to interactions with the election rules.

For Australian Senate elections, a key factor is the formality requirements. BTL votes have more stringent requirements, which ends up creating a systematic bias against BTL votes in the presence of random errors. Candidates who rely on BTL votes (e.g. if they are relying on their individual popularity) will be more affected by random errors than those relying on ATL votes (e.g. via membership of their party). Changing the formality requirements to reduce the disparity between ATL and BTL votes also reduces this bias.

Candidates who rely on accumulating later preferences are more affected by random errors than candidates who rely primarily on their first-preference votes. However, this effect was much weaker than the bias induced by differences in formality requirements.

These results raise questions about how formality rules should be specified in order to be fair to candidates with different voting patterns. More relaxed formality rules could be applied which are less likely to have strong differences across different kinds of votes. For example, a BTL vote could be formal if the first 6 most preferred candidates are clear, even if they are not numbered from 1 to 6 , e.g. a vote with preferences $1,2,4$, $5,6,7$ and no preference 3 still gives a clear ranking of the first 6 candidates.

In this paper we consider only Australian Senate elections with their particular ATL/BTL voting mechanism. Two lessons can be taken from this exercise to other forms of voting. 
First, if there are two or more forms of ballot and the rules for formality are different for these different forms of ballot, then random errors may affect the different forms differently, regardless of whether the voter can choose their form or different voters are assigned to different forms. This is applicable to any kind of election whether plurality voting or ranked voting. Second, considering elections where voters rank candidates with only one form of ballot, e.g. standard STV, Borda, or Condorcet elections, assuming the rules of formality are such that the ballot is truncated when the ranking becomes uninterpretable, then candidates relying on accumulating later preferences will be more affected by random errors than other candidates. But we do not have a real world case that illustrates that truncation errors alone lead to a change in a result.

Acknowledgements. We would like to thank our colleagues who participated in our informal experiment during the 2019 Australian federal election. Thanks also to Philip Stark for very valuable suggestions on improving the paper.

\section{References}

1. Dash, K.S., Puhan, N.B., Panda, G.: Unconstrained handwritten digit recognition using perceptual shape primitives. Pattern Analysis and Applications 21, 413-436 (2018)

2. Richey, S.: Random and systematic error in voting in presidential elections. Political Research Quarterly 66(3), 645-657 (2013), http: / / www. jstor.org/stable/23563171

3. Toghi, B., Grover, D.: MNIST dataset classification utilizing k-NN classifier with modified sliding window metric. CoRR abs/1809.06846 (2018), http: / / arxiv.org/abs / 1809.06846 